\title{
Specific Charge Control for Micro/Nano-Particle Electrostatic Propulsion
}

\author{
Thomas M. Liu ${ }^{*}$, Gregory L. Wagner ${ }^{\dagger}$, Alec D. Gallimore ${ }^{\ddagger}$, and Brian E. Gilchrist ${ }^{\S}$, \\ University of Michigan, Ann Arbor, MI, 48109 \\ and \\ Peter Y. Peterson ${ }^{* *}$ \\ ElectroDynamic Applications, Inc., Ann Arbor, MI, 48105
}

\begin{abstract}
Contact charging of micro- and nano-particles affords the Nanoparticle Field Extraction Thruster (NanoFET) flexibility to adjust its propellant's specific charge. This flexibility permits NanoFET to tune its propulsive performance by adjusting the charging electric fields and acceleration potential. Inherent to the NanoFET design are two features that govern the range of achievable specific charges: the gate electrode configuration (which sets the charging electric fields and the resulting particle charge) and the particle mass density. Electrostatic simulations for various gate electrode configurations were conducted in COMSOL Multiphysics ${ }^{\circledR}$ to provide general scaling relations for the acquired particle charge as a function of gate geometry (i.e., gate orifice diameter $D_{G}$ and inter-electrode gap $H$ ) and particle diameter $d$. The simulation results suggest that configurations with smaller gate aspect ratios $\left(D_{G} / H\right)$ provide greater charging and that the charging behavior is consistent until $d / H$ is large and approaches unity. The benefits to performance and operations of using low density, and particularly hollow, particles were examined. Compared to solid gold particles, hollow ceramic particles with shell thicknesses that are $5 \%$ and $1 \%$ of their diameters have increased specific impulses of $5.3 \mathrm{X}$ and $11.5 \mathrm{X}$, respectively. The use of low mass density or hollow particles gives NanoFET the potential for moderate specific impulse and high thrust-to-power performance.
\end{abstract}

\footnotetext{
* Graduate Student, Aerospace Engineering, liutm@umich.edu, AIAA Student Member

†Undergraduate Student, Aerospace Engineering, greglw@umich.edu, AIAA Student Member

* Arthur F. Thurnau Professor, Aerospace Engineering \& Applied Physics, alec.gallimore@umich.edu, AIAA Associate Fellow

$\S$ Professor, Electrical Engineering \& Space Systems, gilchrst@umich.edu, AIAA Associate Fellow

** Director of Research, peterson@edapplications.com, AIAA Member
} 


\section{Nomenclature}

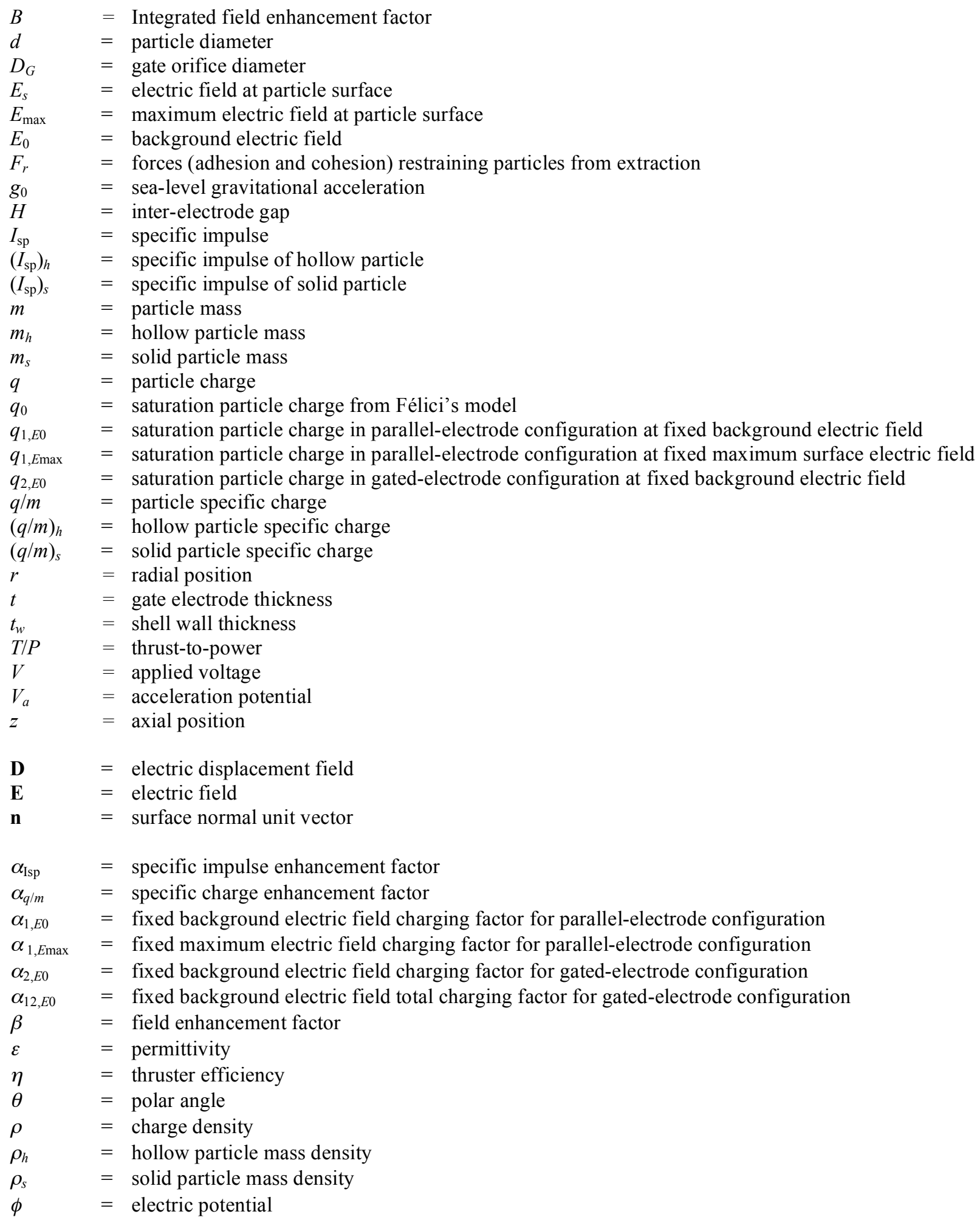




\section{Introduction}

$\mathrm{T}$ RENDS in micro- and nano- in-space propulsion systems are making the logical progression towards devices with high efficiency, variable thrust and specific impulse $\left(I_{\mathrm{sp}}\right)$, small footprints, and low mass to enable deployment of ever smaller and more versatile fleets of spacecraft. The Nanoparticle Field Extraction Thruster (NanoFET) is one such propulsion technology being developed for pico- to small satellite applications.

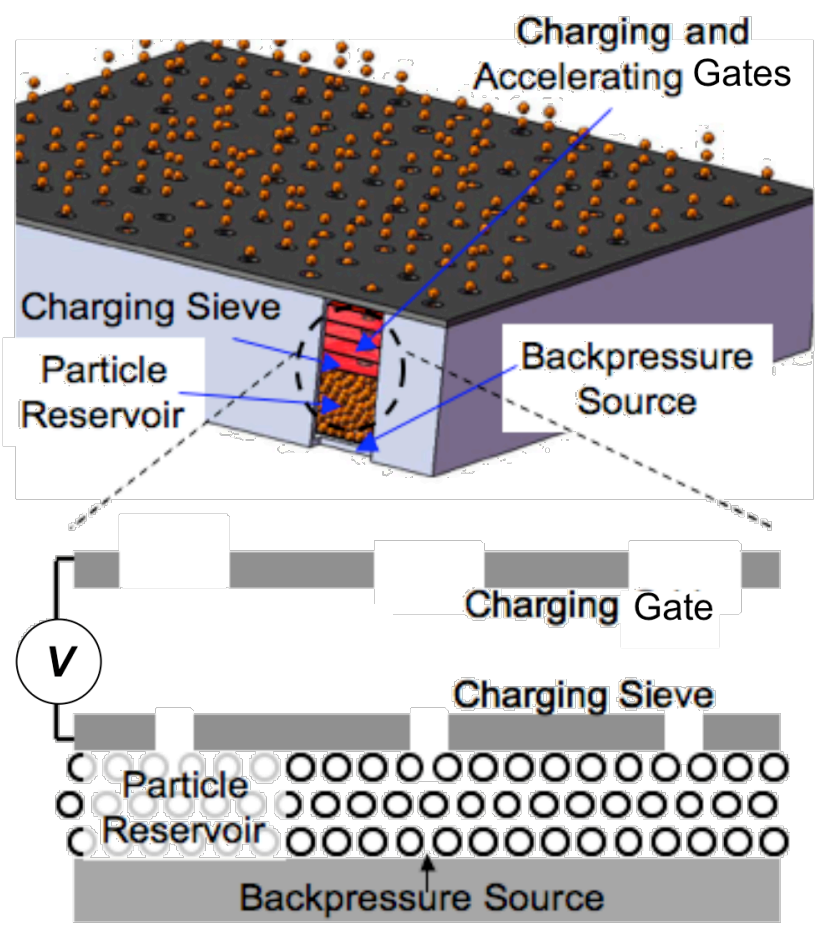

Figure 1. Concept views of NanoFET. Multiple electrode gates may be used to decouple the particle charging and accelerating stages or to achieve higher acceleration potentials.

While similar in operation to colloid thrusters, NanoFET does not rely on droplet formation and extraction as the source of propellant. Instead, the NanoFET system electrostatically charges and accelerates pre-fabricated, solid micro- and nano-particles. As shown conceptually in Figure 1, backpressure feeds the particle propellant in dry powder form towards the charging sieve. There, particle aggregates are dispersed upon passage through the sieve with the aid of piezoelectric-induced inertial forces. Individual particles undergo contact charging and are subsequently accelerated by the electric fields generated by stacked electrode gates. By using micro/nano-electromechanical systems (MEMS/NEMS) fabrication processes, NanoFET achieves a compact, flat-panel configuration that is potentially scalable for a variety of mission needs. ${ }^{1}$

With the propellant electrostatically charged rather than ionized, NanoFET can tune the propellant's charge state by adjusting the charging electric field. Along with the potential to select propellant particle sizes, geometries, and material properties, NanoFET achieves flexibility in adjusting the propellant's specific charge.

NanoFET thus is a variable- $I_{\text {sp }}$ thruster whose performance can be optimized at each point during an orbit maneuver to minimize propellant use or trip time or to accomplish dynamic retasking of the host spacecraft. ${ }^{2}$

The following sections examine two design factors that affect NanoFET propellant's specific charge: the gate electrode geometry and the particle mass density, particularly in the case of hollow particles. Performance projections are also included to illustrate the impact of tunable specific charge on propulsion capabilities.

\section{Particle Contact Charging in Gated-Electrode Configurations}

Félici's analytical model for the saturation charge $q_{0}$ acquired by an isolated, spherical particle of diameter $d$ in contact with a charging electrode and exposed to a uniform electric field $E_{0}$ (in the absence of particle focusing and edge effects) is

$$
q_{0}=\frac{\pi^{3}}{6} \varepsilon E_{0} d^{2}
$$

where $\varepsilon$ is the permittivity of the medium about the particle. ${ }^{3}$ This model has been experimentally validated via dynamic and current measurements of particles (albeit at larger sizes than proposed for NanoFET) charged in a parallel-electrode configuration (Figure 2). ${ }^{4,5}$ In these studies, a particle lifts off the bottom electrode and moves towards the upper electrode if the electrostatic force acting on the charged particle is greater than the restraining forces. Upon contact with the upper electrode, the particle is charged opposite its initial polarity and is directed back towards the bottom electrode. The sequence then repeats, resulting in particle oscillations between the plates. 
Félici's model is valid for the parallel-electrode configuration provided that the particles are small compared to the inter-electrode gap $(d \ll H)$, the particles achieve saturation charging prior to liftoff, and the time scale associated with particle charge transfer to the surrounding medium is large compared to the oscillation period.

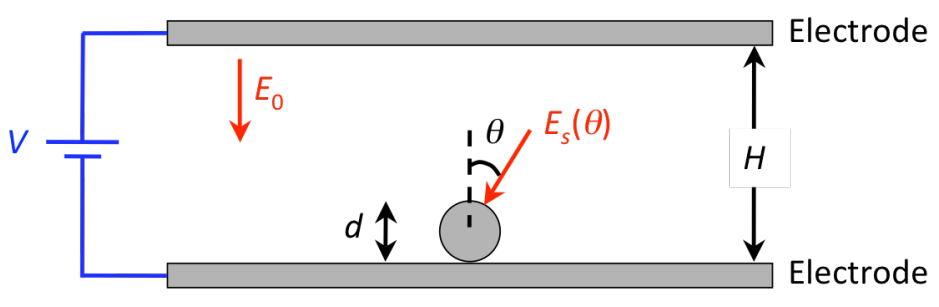

Figure 2. Particle charging in parallel-electrode configuration. Electrode edge effects on the particle are assumed to be negligible.

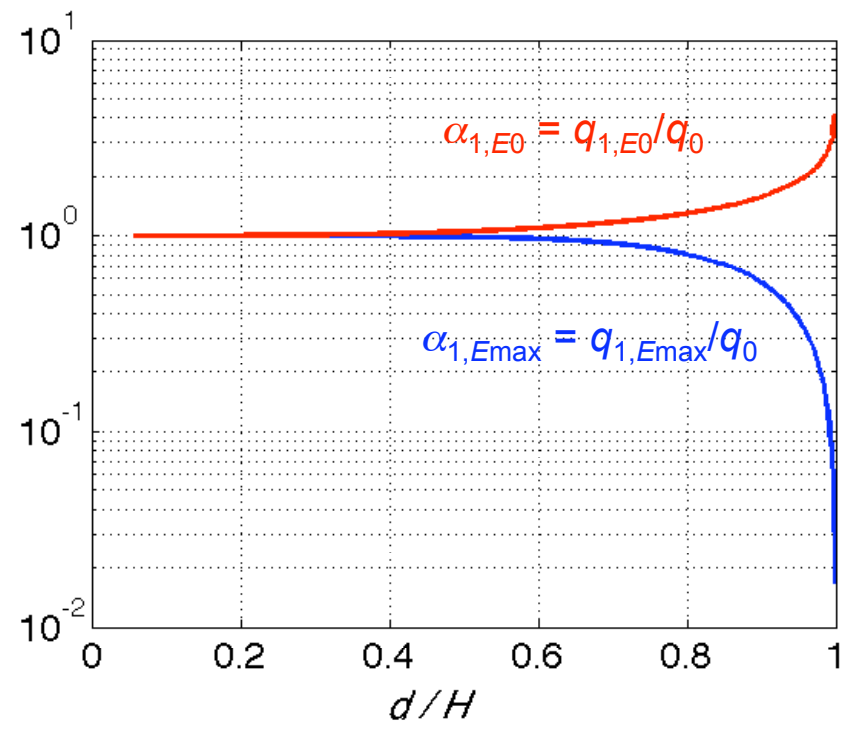

Figure 3. Charging factors for particle charging in parallelelectrode configuration. A unity charging factor exactly agrees with Félici's model (Equation 1).

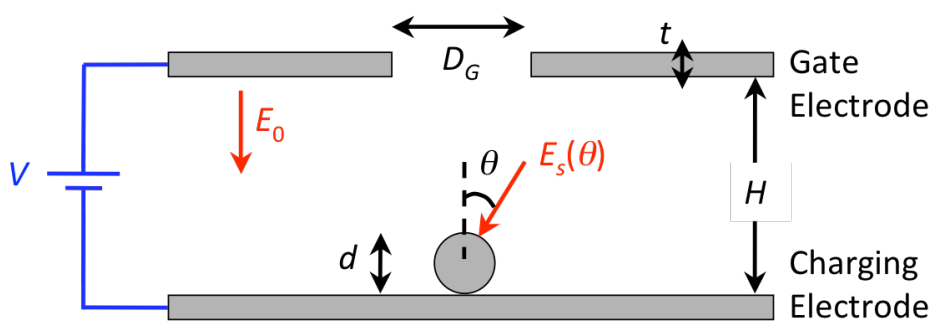

Figure 4. Particle charging in gated-electrode configuration. Particle extraction is possible for $d / D_{G}<1$. The gate orifice is assumed to be centered about the particle.
As $d / H$ increases, the particle field enhancement factor

$$
\beta(\theta) \equiv \frac{E_{s}(\theta)}{E_{0}}
$$

also increases, where $E_{0} \equiv V / H$. For a constant $E_{0}$, which must be no more than $60 \%$ of the field emission/evaporation limit ${ }^{6}$, the resulting particle charge $q_{1, E 0}$ is greater than what is expected from Félici's model by the charging factor (fixed background electric field)

$$
\alpha_{1, E 0} \equiv \frac{q_{1, E 0}}{q_{0}} .
$$

An alternative condition fixes the maximum electric field $E_{\max }=E_{s}(\theta=0)$. For NanoFET, this maximum electric field must be less than the field emission/evaporation limit. Musinski showed that constraining the electric field at the tip of the particle, where field focusing is most significant, results in a charging factor (fixed maximum electric field)

$$
\alpha_{1, E \max } \equiv \frac{q_{1, E \max }}{q_{0}},
$$

which decreases from unity as $d / H$ increases. ${ }^{7}$

Figure 3 summarizes the behavior of both charging factors using the numerical simulation methodology described later in the paper. For cases of constant $E_{0}$ and constant $E_{\max }$, the particle charge deviates less than $5 \%$ from Félici's model for $d / H<$ 0.5 and $d / H<0.64$, respectively. In general, particles charging in a parallelelectrode configuration are thus well described by Equation 1 for $d / H<0.5$.

In order to generate thrust with the charged particles, the upper electrode in Figure 2 must be replaced with a gate electrode that permits particles to leave the system. This gated-electrode configuration is shown in Figure 4, with the gate orifice diameter $D_{G}$ being greater than the particle diameter $d$ to permit particle extraction. 


\section{A. Simulation Methodology}

Electrostatic simulations were conducted in COMSOL Multiphysics $\AA$, a commercial finite element analysis software package, to investigate the impact of the gated-electrode configuration on particle charging. COMSOL's electrostatic application mode, which solves Poisson's equation $\left(\nabla^{2} \phi=-\rho / \varepsilon\right)$, has been previously used in numerical studies of particle contact charging with good agreement with analytical models. ${ }^{8,9}$ For this paper, the geometries considered were not intended to be high-fidelity representations of NanoFET. Instead, simplified models were used to provide insights into scaling relations to aid optimization of gate designs for NanoFET. As such, whereas NanoFET may have multiple gate electrodes, only the first gate is modeled as it supplies the particle charging electric fields; subsequent gates supply inter-gate electric fields to accelerate particle propellant after it has already been charged and extracted. Further, NanoFET's charging sieve is not modeled; instead, as with the parallelelectrode configuration, particles are assumed to be in contact with a continuous charging electrode. Future work is intended to refine the simulations and explore sieve configuration effects on particle charging.
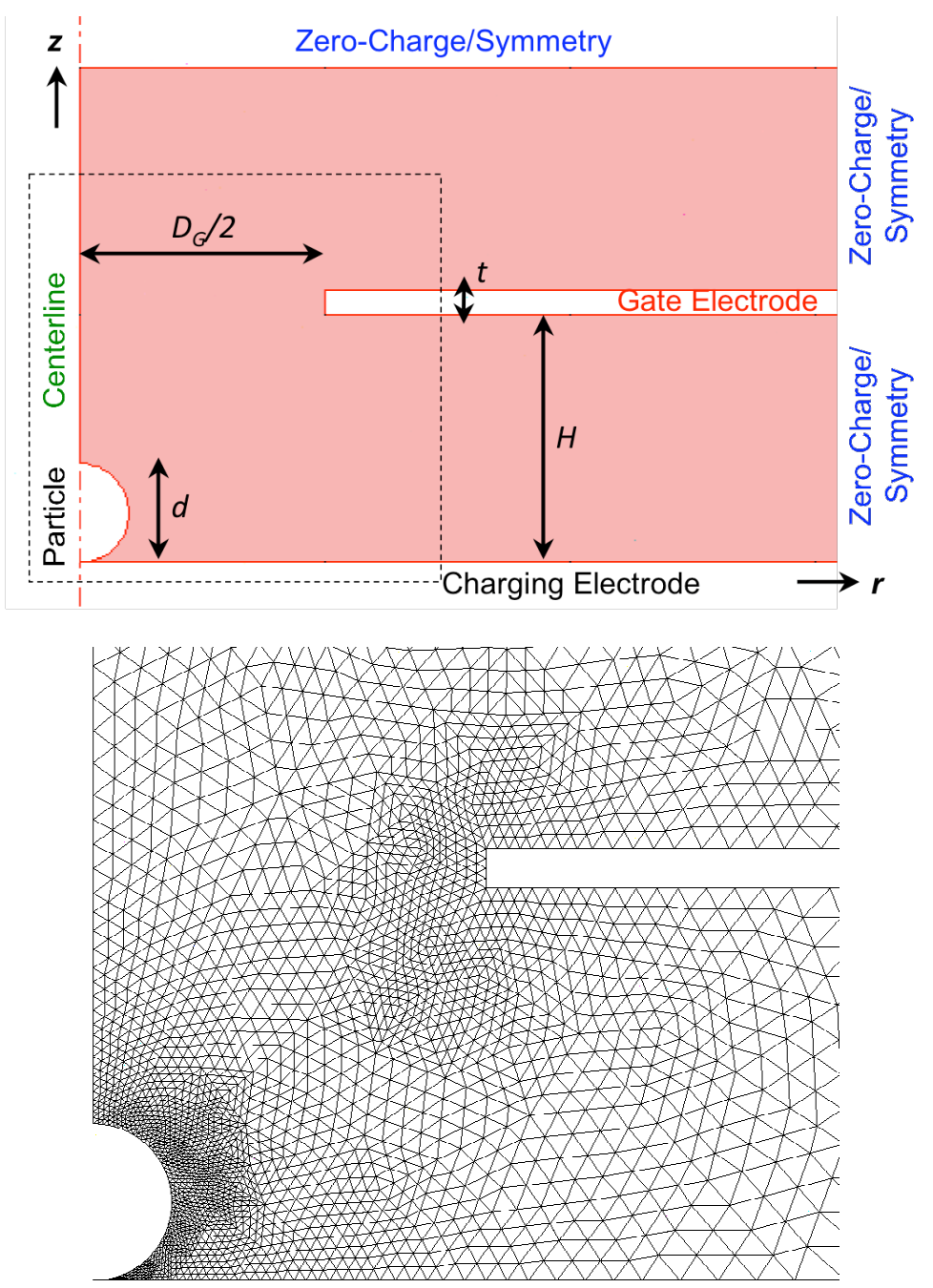

Figure 5. COMSOL simulation domain for gated-electrode configuration. (Top) Boundary conditions shown in $r$-z space (not to scale). (Bottom) Representative unstructured mesh shown for highlighted region in top figure.
As shown in Figure 5, the simulation domain is axi-symmetric ( $r-z$ space $)$ assuming the gate orifice is centered about the particle; $r=0$ is defined as the axis of symmetry (centerline). A gate electrode of thickness $t=0.1 \mathrm{~mm}$ is modeled as an equipotential region of bias $V$ placed $1 \mathrm{~mm}$ above a grounded charging electrode. These dimensions are not critical, since the simulation results are non-dimensionalized and scalable. Zero-charge/symmetry $(\mathbf{n} \cdot \mathbf{D}$ $=0$ ) boundary conditions are in place at the top and right $(z=20 \mathrm{~mm}$ and $r=20 \mathrm{~mm}$, respectively) boundaries of the simulation domain. The medium surrounding the particle (shaded region in Figure 5) has free-space permittivity, and the rigid, isolated, and grounded particle contacts the bottom electrode at the $r-z$ space's origin.

As shown in Figure 5, an unstructured mesh of triangular elements is used for the simulation domain. Using COMSOL, the electric field at the particle surface can be mapped. Applying Gauss's law $(q=$ $\oiint \varepsilon \mathbf{E} \bullet d \mathbf{A}$ ) for the particle and utilizing azimuthal symmetry, the saturation charge $q$ on the particle is

$$
q=\frac{\pi}{2} \varepsilon E_{0} d^{2} B
$$

where the integrated field enhancement factor $B$ is defined as

$$
B \equiv \int_{0}^{\pi} \beta(\theta) \sin \theta d \theta
$$

This formulation implicitly assumes that the particle charges classically and that quantum effects are not significant. Note that Félici's model corresponds to $B=\pi^{2} / 3$. 


\section{B. Constant Background Electric Field/Applied Voltage Results}

A constant background electric field $E_{0}=V / H=10 \mathrm{~V} / \mu \mathrm{m}$ was used to generate the results below. Since the inter-electrode spacing is fixed $(H=1 \mathrm{~mm})$ in the simulation domain, this simulation case also represents the application of a constant bias voltage $(V=10 \mathrm{kV})$ in the simulation. Figure 6 shows representative electric field mapping results for various $d / H$.
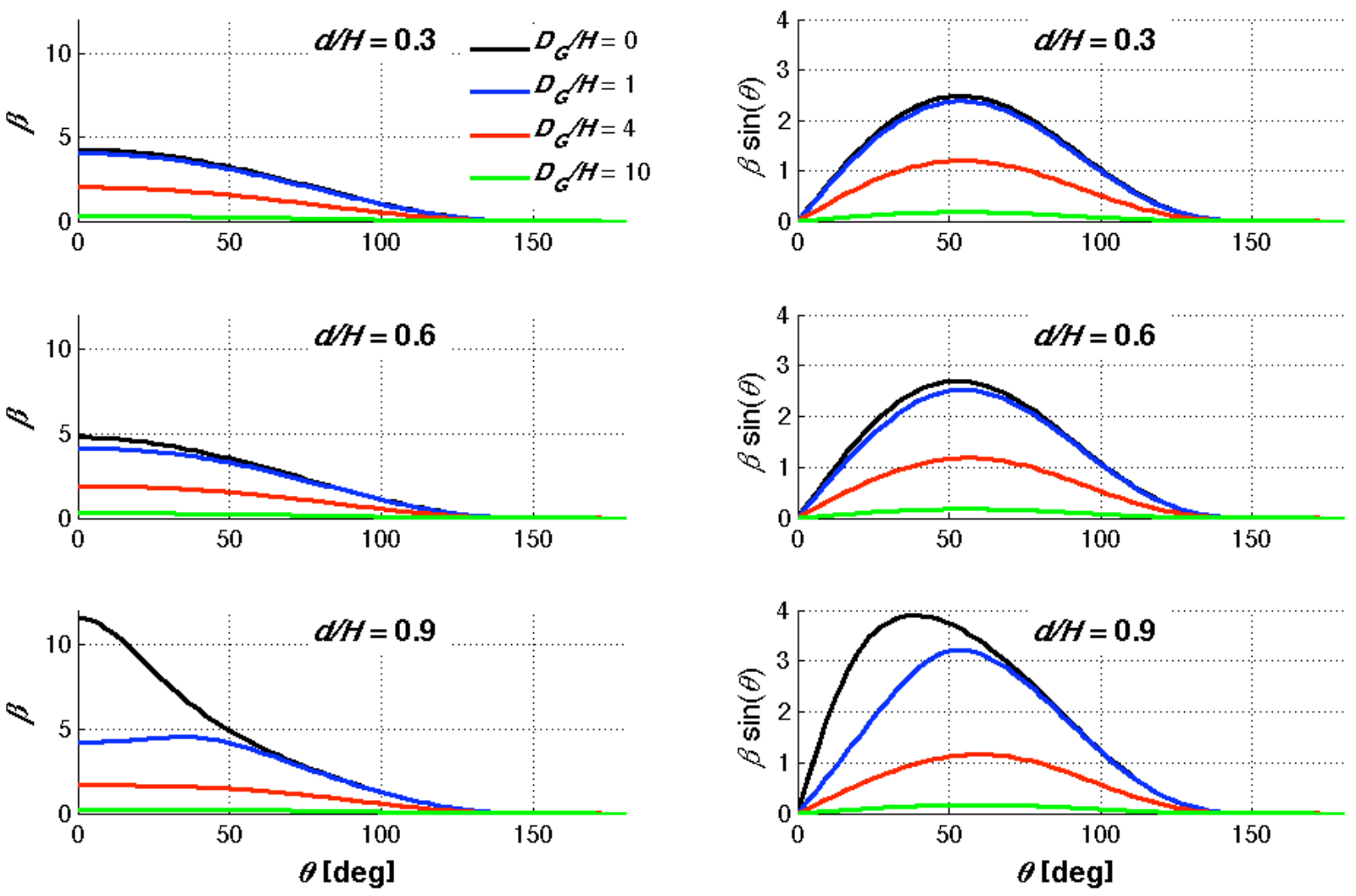

Figure 6. Electric field behavior at particle surface for gated-electrode configuration. Field enhancement factor $\beta$ is proportional to the surface charge density. The area under the $\beta \sin \theta$ curve is proportional to the particle charge for a given particle size.

In general, the peak electric field occurs at the particle tip $(\theta=0)$. Exceptions occur for $d / H>0.6$ and $D_{G} / H<3$, where the edges of the gate orifice approach the particle surface and effects due to the gate thickness are apparent. At a given $d / H$, both the surface electric field and the integrated field enhancement factor decrease as the gate orifice diameter increases. For a given gate aspect ratio (i.e., constant $D_{G} / H$ ), larger particles (i.e., larger $d / H$ ) result in higher peak surface electric fields and larger integrated field enhancement factors. Note that for cases where $D_{G} / H=$ 0 , thus recovering the parallel-electrode configuration, the calculated particle charges agree well with Félici's model for small $d / H$. For example, at $d / H=0.1$ and $d / H=0.3$, the calculated charges deviate from Félici's model by only $0.03 \%$ and $1 \%$, respectively, which serves to validate the simulations.

The presence of the gate orifice results in a particle charge $q_{2, E 0}<q_{1, E 0}$, the particle charge with no gate orifice. This decrease in contact charging is represented by the charging factor (fixed background electric field) shown in Figure 7:

$$
\alpha_{2, E 0} \equiv \frac{q_{2, E 0}}{q_{1, E 0}} .
$$




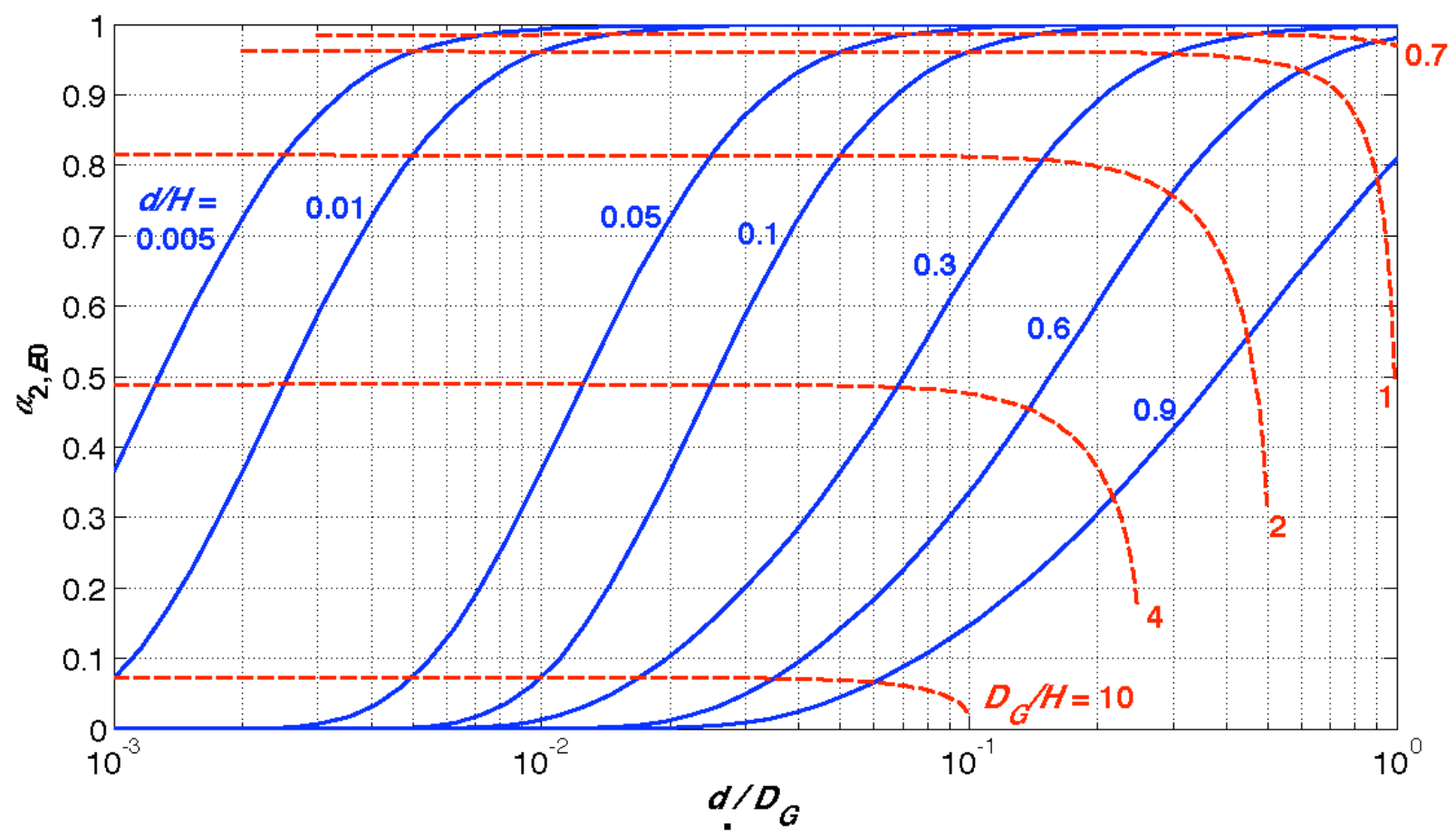

Figure 7. Charging factor for gated-electrode configuration. Particle extraction is possible for $d / D_{G}<1$.

As $d / D_{G}$ increases, the charging factor $\alpha_{2, E 0}$ approaches unity, thus recovering the parallel-electrode configuration. At a given particle size and $H, \alpha_{2, E 0}$ decreases with larger gate orifice diameters; at a given particle size and $D_{G}, \alpha_{2, E 0}$ increases with larger inter-electrode gaps. Thus, for a given particle size, a smaller gate aspect ratio $D_{G} / H$ provides a greater charging factor. For example, $\alpha_{2, E 0}>0.96$ for $D_{G} / H<1$ (and $d / H<0.3$ ). Note that for a fixed gate configuration (i.e., both $D_{G}$ and $H$ are fixed), two distinct regimes exist for the charging factor's behavior. The charging factor remains essentially constant for small particles and decreases rapidly for $d / H>0.6$.

The saturation charge for a particle in the gated electrode configuration is thus

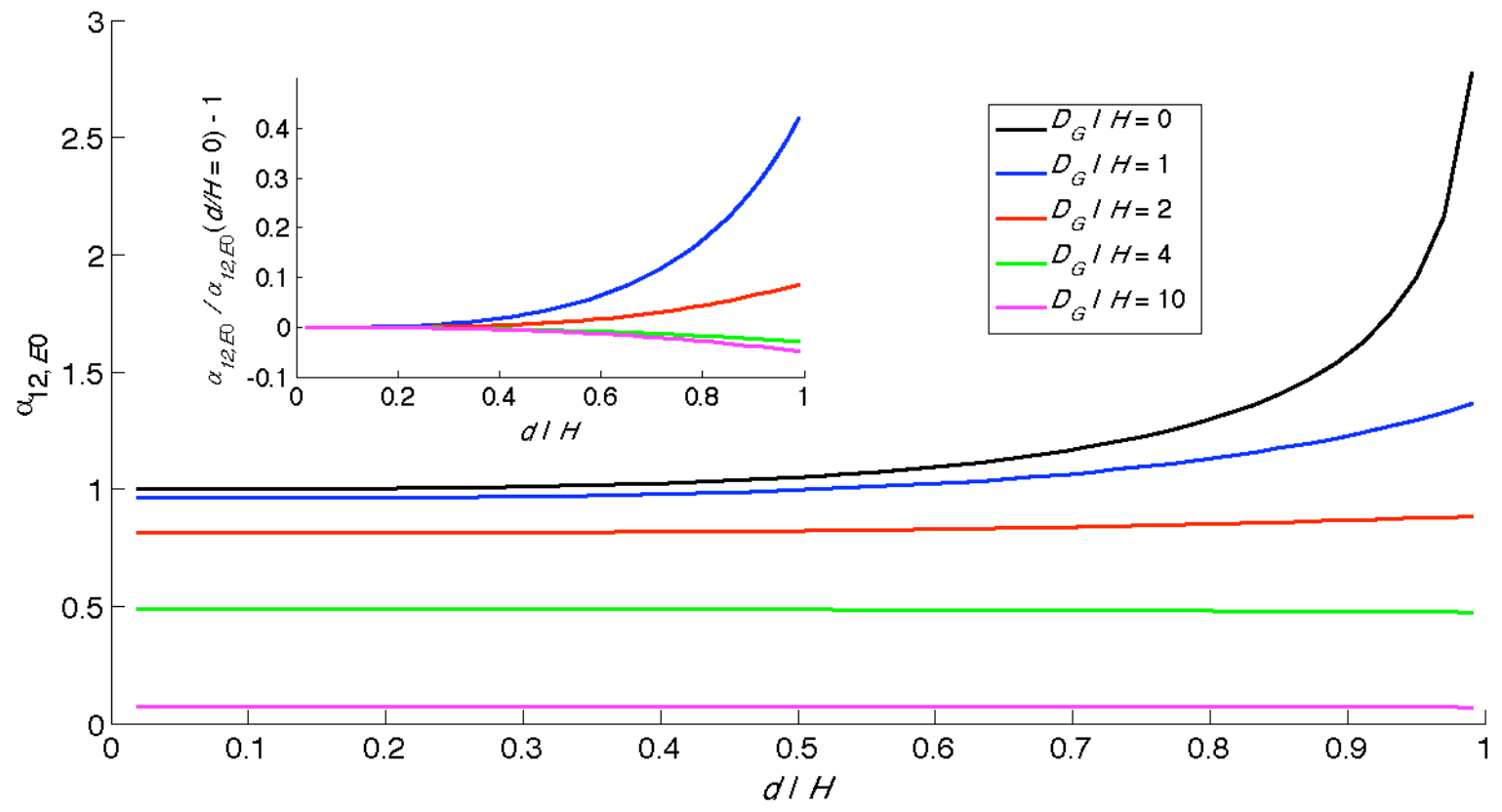

Figure 8. Total charging factor for gated-electrode configuration. Inset figure shows the total charging factor's deviation from its value at $d / H=0$.

American Institute of Aeronautics and Astronautics 092407 


$$
q_{2, E 0}=\alpha_{1, E 0} \alpha_{2, E 0} q_{0}=\alpha_{12, E 0} q_{0}
$$

where $\alpha_{12, E 0}$ is the total charging factor for gated-electrode configurations. As the gate aspect ratio $D_{G} / H$ increases, $\alpha_{12, E 0}$ decreases; equivalently, for a given $d$ and $H, \alpha_{12, E 0}$ decreases for larger gate orifice diameters. In Figure 8, $\alpha_{12, E 0}$ increases with $d / H$ for $D_{G} / H$ values of 0,1 , and 2 such that for a fixed gate configuration (i.e., both $D_{G}$ and $H$ are fixed), increasing the particle size increases the total charging factor. In the case $D_{G} / H=0$, recovery of the parallel-electrode configuration means that $\alpha_{12, E 0}=\alpha_{1, E 0}$. For $D_{G} / H$ values of 4 and 10 , the total charging factor is insensitive to $d / H$ but has negative concavity; thus, for these cases of fixed gate configuration, $\alpha_{12, E 0}$ slightly decreases with larger particle sizes.

Because the above results were obtained under constant $E_{0}$ conditions, caution is needed when applying results for large $d / H$ and small $D_{G} / H$ (or equivalently, large $d / D_{G}$ ). As can be seen in Figure 6 , the large field enhancement in these cases may result in electric fields that exceed the field emission/evaporation limit. Future work will examine how the charging factors behave for conditions in which the maximum allowable electric field is fixed.

\section{Use of Low Mass Density Particles}

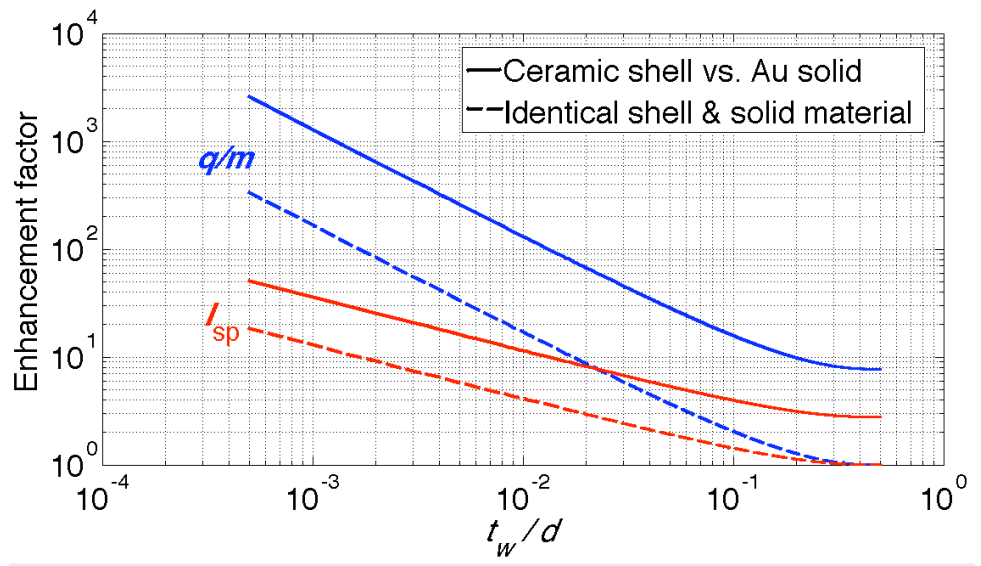

Figure 9. Enhancement of specific charge and specific impulse for hollow compared to solid particles. Ceramic shells $\left(\rho_{h}=2.5\right.$ $\left.\mathrm{g} / \mathrm{cm}^{3}\right)$ are assumed to be either semiconducting or to have a thin metal coating for contact charging.

As shown in Equation 1 for contact charging, particles of the same size acquire the same charge for a given electric field. A particle with lower mass density, therefore, would have less mass than its higher mass density counterpart, thus resulting in a higher specific charge (charge-to-mass ratio) $q / m$. Since $I_{\mathrm{sp}}=$ $g_{0}{ }^{-1}\left(2 q V_{\mathrm{a}} / m\right)^{1 / 2}$, the use of lower mass density particles permits higher $I_{\mathrm{sp}}$ to be achieved for a given particle size and acceleration potential $V_{a}$. Particles of lower mass density also permit lower acceleration potentials to be used to meet a given $I_{\mathrm{sp}}$ target; this reduction in operating voltages could simplify power system designs and improve operational reliability.

\section{A. Hollow Particles}

Lower mass density may also be achieved by using hollow rather than solid particles. The specific charge enhancement factor $\alpha_{q / m}$ for a hollow particle of shell wall thickness $t_{w}$ compared to a solid particle of the same diameter $d$ is

$$
\alpha_{q / m} \equiv \frac{(q / m)_{h}}{(q / m)_{s}}=\frac{m_{s}}{m_{h}}=\frac{\rho_{s}}{\rho_{h}}\left[1-\left(1-2 \frac{t_{w}}{d}\right)^{3}\right]^{-1},
$$

where $\rho_{s}$ and $\rho_{h}$ refer to the mass densities of the solid particle and the shell material, respectively. For $t_{w} \ll d$, the specific charge enhancement factor can be approximated as $\alpha_{q / m} \approx\left(6 t_{w} / d\right)^{-1}$, which is within $10 \%$ of the true value for $t / d<0.05$. The corresponding specific impulse enhancement factor for a fixed acceleration potential is

$$
\alpha_{\mathrm{Isp}} \equiv \frac{\left(I_{\mathrm{sp}}\right)_{h}}{\left(I_{\mathrm{sp}}\right)_{s}}=\sqrt{\alpha_{\mathrm{q} / \mathrm{m}}}
$$

Both enhancement factors are shown in Figure 9. In the case of dissimilar materials between the shell and solid particles, gold solids and ceramic shells are chosen to illustrate the enhancement. For this case study, a hollow 
particle whose wall thickness is $5 \%$ of its diameter displays an $I_{\text {sp }}$ improvement of $5.3 \mathrm{X}$. If the hollow particle instead has a wall thickness that is $1 \%$ of the particle diameter, then the $I_{\mathrm{sp}}$ improvement increases to a factor of $11.5 \mathrm{X}$.

\section{B. Performance Projections}

Figure 10 shows projections for NanoFET's achievable $I_{\mathrm{sp}}$ and thrust-to-power for various propellant types. Particles are assumed to undergo classical inductive charging in $100-\mathrm{V} / \mu \mathrm{m}$ background electric fields in gate geometries to remain below the electron field emission limit $(\sim 1000 \mathrm{~V} / \mu \mathrm{m})$. For a $10-\mathrm{kV}$ acceleration potential, the use of solid or hollow ceramic (metallized ${ }^{10}$ or semiconductor ${ }^{11}$ ) particles (with sufficient electrical conductivity due to inherent semiconductivity or metallization), can achieve specific impulses of hundreds of seconds for particles on the order of tens of nanometers in diameter. Metal shell particles ${ }^{12}$ may also achieve these $I_{\text {sp }}$ values for sufficiently thin shells; for example, a hollow gold particle of shell wall thickness that is $2 \%$ of its diameter would have the same performance as the solid ceramic particle. Such moderate $I_{\mathrm{sp}}$ values result in high thrust-to-power $\left(T / P=2 \eta / g_{0} I_{\mathrm{sp}}\right)$ performance projections for NanoFET, with possible $T / P$ in excess of $100 \mathrm{mN} / \mathrm{kW}$.
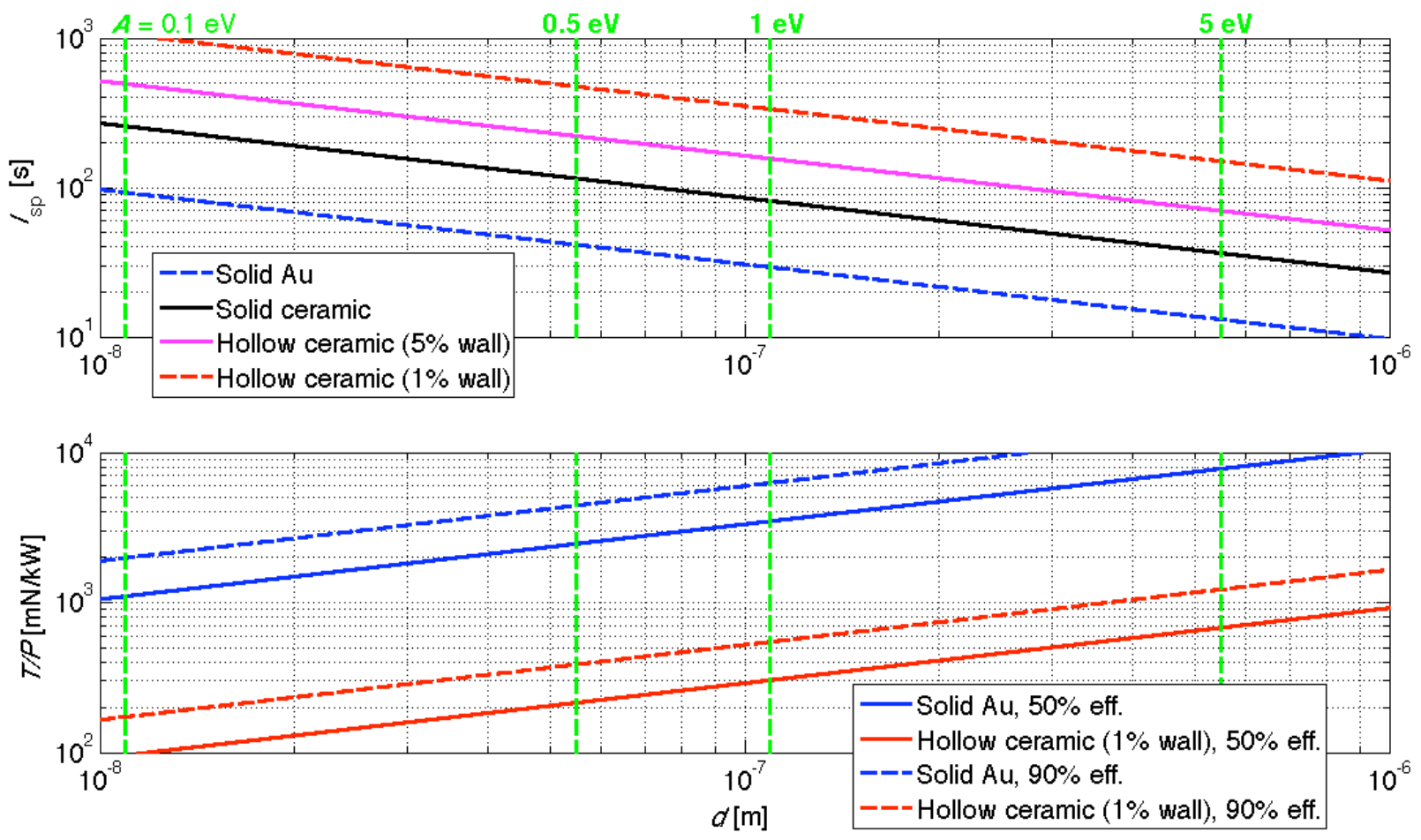

Figure 10. Projected NanoFET performance for $100-\mathrm{V} / \mu \mathrm{m}$ charging electric field, $10-\mathrm{kV}$ acceleration potential, and unity total charging factor. $2.5 \mathrm{~g} / \mathrm{cm}^{3}$ is used as the mass density of ceramic materials, which are assumed to be either semiconducting or to have a thin metal coating for contact charging. Hollow particles with shell wall thicknesses that are 1\% and 5\% of the particle diameters are shown along with 50\% and $90 \%$ thruster efficiency reference points. The Hamaker constant (A) reference lines designate particle extraction thresholds.

For the particles to be extracted after charging, the adhesive and cohesive forces $F_{r}$ holding the particles to the charging electrode and to each other, respectively, must be overcome. These forces scale as $F_{r} \propto A d$, where $A$ is the Hamaker constant of the two contacting materials. ${ }^{13}$ In Figure 10, the Hamaker constant reference lines designate the smallest particle that can be extracted by the charging electric field for a given Hamaker constant; for example, if the Hamaker constant is $1 \mathrm{eV}$, particles smaller than $100 \mathrm{~nm}$ could not be extracted unless an electric field greater than $100 \mathrm{~V} / \mu \mathrm{m}$ or additional energy input (such as from inertial forces) are supplied. Treating and functionalizing particle surfaces to modify their effective Hamaker constants and reduce adhesive and cohesive forces is one approach to enable the use of smaller particles in NanoFET. However, another approach becomes possible with the use of lower density and hollow particles, in which larger particles could be used to meet a given $I_{\text {sp }}$ target. Because electrostatic forces in contact charging scale quadratically with particle diameter, particle adhesion and cohesion 
issues are less significant for larger particles, thus improving the propellant's dry storage capability and reducing the risk of feed system jamming in NanoFET.

\section{Conclusion}

During operations, NanoFET may control its propellant's specific charge by adjusting the charging electric field. But the gate configuration design (affects particle charge) and propellant selection (affects particle mass) determine the range of achievable specific charge. A greater specific charge range, resulting in greater flexibility for a variable- $I_{\mathrm{sp}}$ thruster, is accomplished using gate configurations with small gate aspect ratios $\left(D_{G} / H \leq 1\right.$ recommended). The use of lower mass density materials compared to metals or the use of hollow particle propellant also increases the range of achievable specific charge.

\section{Acknowledgments}

The work presented was funded in part by an Air Force Office of Scientific Research Phase 2 STTR (FA955009-C-0079, managed by Dr. Mitat Birkan) and a National Science Foundation Graduate Research Fellowship (Liu). Thanks to Harvey Elliott, Shane Moore, Greg Salvesen, and Yuly Wung at the University of Michigan for their initial studies of particle contact charging using COMSOL.

\section{References}

1 Massey, D., Musinski, L., Liu, T., Gallimore, A., and Gilchrist, B., "The Nanoparticle Field Extraction Thruster: NanoFET," JANNAF 3rd Spacecraft Propulsion Subcommittee Meeting, Orlando, FL, 8-12 December 2008.

2 Liu, T., Musinski, L., Patel, P., Gallimore, A., Gilchrist, B., and Keidar, M., "Nanoparticle electric propulsion for space exploration," in Space Technology and Applications International Forum - STAIF 2007, edited by M. S. El-Genk, American Institute of Physics, Albuquerque, NM, 2007, pp. 787-94.

${ }^{3}$ Félici, N.-J., "Forces et charges de petits objets en contact avec une électrode affectée d'un champ électrique," Revue Générale de l'Électricité, 75 (1966), pp. 1145-60.

${ }^{4}$ Tobazéon, R., "Electrohydrodynamic behaviour of single spherical or cylindrical conducting particles in an insulating liquid subjected to a uniform DC field," J. Phys. D: Appl. Phys., 29 (1996), pp. 2595-608.

${ }_{5}$ Musinski, L., Liu, T., Gilchrist, B., and Gallimore, A., "Electrostatic charging of micro- and nano-particles for use with highly energetic applications," Journal of Electrostatics, 4 November 2008, pp. 1-8.

${ }^{6}$ Trottenberg, T., Kersten, H., and Neumann, H., "Feasibility of electrostatic microparticle propulsion," New Journal of Physics, 10 (2008).

${ }^{7}$ Musinski, L., "Investigation of a micro- and nano-particle in-space electrostatic propulsion concept," Ph.D. dissertation, University of Michigan, 2009.

${ }^{8}$ Nader, B.F., Castle, G.S.P., and Adamiak, K., "Effect of surface conduction on the dynamics of induction charging of particles," J. Electrostatics, 67 (2009), pp. 394-9.

${ }^{9} \mathrm{Yu}$, D., Castle, G.S.P., and Adamiak, K., "The effect of shape and roughness on the maximum induction charge for small particles," J. Phys.: Conf. Ser., 142 (2008).

${ }^{10}$ Hirsch, L.R., Gobin, A.M., Amanda, R.L., Tam, F., Drezek, R.A., Halas, N.J., and West, J.L., "Metal nanoshells," Annals of Biomedical Engineering, 34 No. 1, (2006), pp. 15-22.

${ }^{11}$ Koktysh, D.S., Liang, X., Yun, B-G., Pastoriza-Santos, I., Matts, R.L., Giersig, M., Serra-Rodríguez, C., Liz-Marzán, L.M., and Kotov, N.A., "Biomaterials by design: layer-by-layer assembled ion-selective and biocompatible films of $\mathrm{TiO}_{2}$ nanoshells for neurochemical monitoring, Advanced Functional Materials, 12 No. 4 (2002), 255-65.

${ }^{12}$ Graf, C. and van Blaaderen, A., "Metallodielectric colloidal core-shell particles for photonic applications," Langmuir, 18 (2002), pp. 524-34.

${ }^{13}$ Bowling, R., “Analysis of particle adhesion on semiconductor surfaces,” J. Electrochem. Soc., 132 (1985), pp. $2208-14$. 